\title{
Enamel and dentine demineralization by a combination of starch and sucrose in a biofilm - caries model
}

\begin{abstract}
Juliana Nunes BOTELHO(a) Mario VILLEGAS-SALINAS(b) Pía TRONCOSO-GAJARDO(b) Rodrigo Andrés GIACAMAN(b) Jaime Aparecido CURY(a)
\end{abstract}

(a) University of Campinas - UNICAMP, Piracicaba Dental School, Department of Physiological Sciences, Piracicaba, SP, Brazil.

(b) University of Talca - UTALCA, Department of Oral Rehabilitation, Cariology Unit, Talca, Región del Maule, Chile.

Declaration of Interests: The authors certify that they have no commercial or associative interest that represents a conflict of interest in connection with the manuscript.

\section{Corresponding Author:}

Jaime Aparecido Cury

E-mail: jcury@unicamp.br

DOI: 10.1590/1807-3107BOR-2016.vol30.0052

Submitted: Sep 14, 2015

Accepted for publication: Jan 11, 2016

Last revision: Feb 17, 2016

\begin{abstract}
Sucrose is the most cariogenic dietary carbohydrate and starch is considered non-cariogenic for enamel and moderately cariogenic for dentine. However, the cariogenicity of the combination of starch and sucrose remains unclear. The aim of this study was to evaluate the effect of this combination on Streptococcus mutans biofilm composition and enamel and dentine demineralization. Biofilms of S. mutans UA159 were grown on saliva-coated enamel and dentine slabs in culture medium containing $10 \%$ saliva. They were exposed (8 times/day) to one of the following treatments: $0.9 \% \mathrm{NaCl}$ (negative control), $1 \%$ starch, $10 \%$ sucrose, or $1 \%$ starch and $10 \%$ sucrose (starch + sucrose). To simulate the effect of human salivary amylase on the starch metabolization, the biofilms were pretreated with saliva before each treatment and saliva was also added to the culture medium. Acidogenicity of the biofilm was estimated by evaluating (2 times/day) the culture medium $\mathrm{pH}$. After 4 (dentine) or 5 (enamel) days of growth, biofilms $(n=9)$ were individually collected, and the biomass, viable microorganism count, and polysaccharide content were quantified. Dentine and enamel demineralization was assessed by determining the percentage of surface hardness loss. Biofilms exposed to starch + sucrose were more acidogenic and caused higher demineralization $(p<0.0001)$ on either enamel or dentine than those exposed to each carbohydrate alone. The findings suggest that starch increases the cariogenic potential of sucrose.
\end{abstract}

Keywords: Amylases; Biofilms; Dental Caries; Dietary Carbohydrates; Tooth Demineralization.

\section{Introduction}

Dental caries is a sugar biofilm-dependent disease, ${ }^{1}$ and sucrose is the most cariogenic dietary carbohydrate. ${ }^{2}$ Starch, a major source of dietary carbohydrate, is considered non- or slightly cariogenic when used as the sole source of dietary carbohydrate. ${ }^{3}$ However, starch is currently consumed simultaneously or interspersed with sucrose, ${ }^{4}$ and this combination could influence the biofilm composition, modulating the pathogenesis of dental caries. ${ }^{5}$

The increased cariogenic potential of this combination of starch and sucrose (starch + sucrose) has been explained by the fact that these two carbohydrates, in the presence of the enzymes salivary 
a-amylase and glycosyltransferases, enhance the formation of highly insoluble extracellular polysaccharides (EPS) and structurally change the biofilm matrix. This would result in the accumulation of strong, cohesive, and adherent biofilms on dental surfaces. ${ }^{5}$ The cariogenic potential of this combination was suggested by in vitro studies evaluating the compositions of Streptococcus mutans biofilms formed on hydroxyapatite discs. ${ }^{6,7}$ Furthermore, starch + sucrose caused a greater number of enamel caries in rats ${ }^{8,9}$ and induced higher in situ demineralization on deciduous ename ${ }^{10}$ than sucrose. However, the greater cariogenicity of starch + sucrose was not confirmed by two subsequent studies, one using a multispecies biofilm model formed on enamel slabs and another evaluating caries in rats. ${ }^{11}$ Moreover, regarding root dentine, starch + sucrose was not significantly more cariogenic than sucrose, when evaluated in situ. ${ }^{12}$

These inconsistencies could be explained by the mechanism of starch hydrolysis in the mouth. Salivary amylase, which is required to metabolize starch, ${ }^{13}$ is responsible for approximately $75 \%$ of the total amylase activity in biofilms. ${ }^{14}$ Therefore, to evaluate the cariogenic potential of starch + sucrose, we used a validated $S$. mutans biofilm mode ${ }^{15}$ that was previously tested to evaluate the cariogenicity of milk..$^{16,17}$ This model was modified by the addition of saliva to simulate the key role of salivary amylase in starch metabolism. This model also simulates the "fast and famine" exposure to dietary sugars to which dental biofilm is subjected in the mouth.

\section{Methodology}

\section{Experimental design}

Independent studies were conducted using slabs of bovine enamel or dentine. S. mutans UA159 biofilms were grown on these slabs using a validated mode ${ }^{15}$ that was modified to simulate the action of salivary amylase. Biofilms were grown in ultrafiltered (10-kDa-cutoff membrane; Prep/Scale; Millipore, Billerica,USA), buffered tryptone-yeast extract broth (UTYEB), and exposed 8 times/day to one of the following treatments: $0.9 \% \mathrm{NaCl}, 1 \%$ starch, $10 \%$ sucrose, and $1 \%$ starch plus $10 \%$ sucrose (starch + sucrose). Each experiment was performed 3 times, each in triplicate $(n=9)$. To simulate the effect of salivary amylase, saliva was added to the culture medium, and the biofilms were also pretreated with saliva before being exposed to the treatments described above. Culture medium was changed two times per day, at the beginning and at the end of the treatments (Figure 1), and its $\mathrm{pH}$ was determined as an indicator of biofilm acidogenicity. After 4 days for dentine and 5 days for enamel, the biomass (dry weight), viable bacteria count, and polysaccharide composition of the biofilm samples were determined. Demineralization induced on enamel and dentine slabs was assessed as the percentage of surface hardness (SH) loss. For statistical analyses, each biofilm/slab was considered as an experimental unit, with the data for enamel and dentine analyzed independently.

\section{Enamel and dentin slabs preparation}

Flattened and polished enamel and root dentine slabs $(4 \times 7 \times 1 \mathrm{~mm})$ were obtained from bovine incisors. ${ }^{15}$ Baseline $\mathrm{SH}$ of the slabs was measured using a Knoop microhardness tester coupled to FM-ARS 900 software (Future-Tech Corp., Kawasaki, Japan). Three indentations, spaced $100 \mu \mathrm{m}$ apart, were made using a load of $50 \mathrm{~g}$ for the enamel and $5 \mathrm{~g}$ for the dentine for 5 seconds. Slabs with $\mathrm{SH}$ $323.1 \pm 8.7$ and $40.5 \pm 2.0 \mathrm{~kg} / \mathrm{mm}^{2}$ for enamel and dentine, respectively, were used in the study, after sterilization with ethylene oxide.

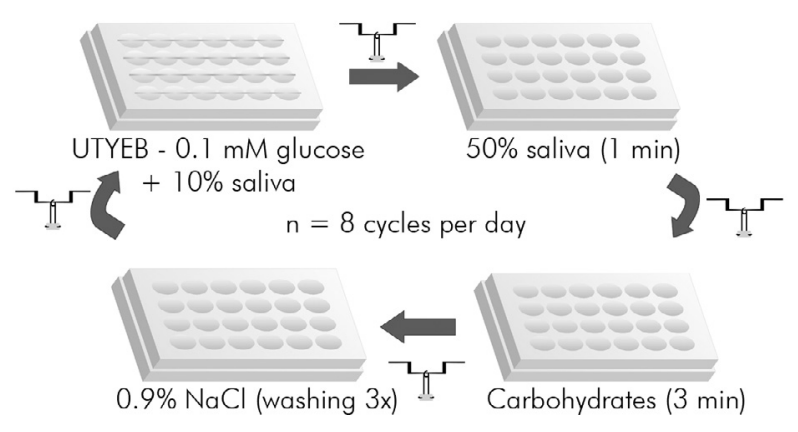

Figure 1. Diagram of the treatments administered 8 times/day $(9: 00,10: 30,12: 00,13: 00,14: 30,16: 00,17: 00$, and $18: 30 \mathrm{~h}$ ) to the biofilms formed on slabs of enamel or dentine $\left(\tau^{-}\right)$. The medium was changed twice/day, at the beginning of the treatments $(9: 00 \mathrm{~h})$ and at the end $(18: 30 \mathrm{~h})$. 


\section{Saliva collection and preparation}

Whole saliva was collected on ice from two healthy volunteers (22 and 24 years old) who chewed paraffin film (Parafilm M; American Can Co., Neenah, USA). They had not used antimicrobials, mouthwashes, or any other medication known to affect salivary composition and flow during the preceding 3 months. Both participants provided written informed consent and the protocols were previously approved by the Research and Ethics Committee of Piracicaba Dental School (Protocol No. 104/2011).

Saliva was used: (1) to form an acquired pellicle on the enamel and dentine surfaces, (2) to pretreat the slabs before treatments, and (3) as an additive to the culture medium in which the biofilms were grown. Saliva collection was performed daily in the morning before any meal and in the afternoon after $2 \mathrm{~h}$ of fasting. For acquired pellicle formation, saliva was diluted 1:1 with adsorption buffer and supplemented with the protease inhibitor phenylmethylsulfonyl fluoride $(1.0 \mathrm{mmol} / \mathrm{L} \text { final concentration })^{18}$ and then centrifuged at $3,800 \mathrm{~g}$ for $10 \mathrm{~min}$ at $4{ }^{\circ} \mathrm{C}$. Saliva used to pretreat the biofilms and that added to culture medium was collected daily and immediately centrifuged as described above. Both supernatants were collected and individually filtered (Filtermax $0.2 \mu \mathrm{m}$ Vacuum System, TPP, St. Louis, USA). The clarified, filter-sterilized saliva was added to the culture medium in a 1:10 (v/v) proportion. Saliva used to pretreat the biofilm was diluted 1:1 (v/v) with $0.9 \% \mathrm{NaCl}$. The amylase activity of the saliva source was assessed using the lugol test (positive after 15 min of incubation).

\section{S. mutans biofilm growth}

For the acquired pellicle formation, slabs were maintained in a 24-well plate and incubated with filtered saliva in an orbital shaker at $60 \mathrm{rpm}$ and $37^{\circ} \mathrm{C}$ for 30 minutes. The slabs coated with human salivary pellicle were individually positioned in a new 24-well plate containing $2.0 \mathrm{ml}$ of $S$. mutans UA159 inoculum (OD 1.6 at $600 \mathrm{~nm}$ ) prepared in a ratio of 1:500 in UTYEB supplemented with $1 \%$ sucrose. After $8 \mathrm{~h}$ at $37^{\circ} \mathrm{C}$ in an atmosphere containing $10 \% \mathrm{CO}_{2}$, the slabs were transferred to another plate where they were immersed in $2.0 \mathrm{~mL}$
UTYEB containing $0.1 \mathrm{mM}$ glucose (basal salivary concentration) and 10\% saliva.

After $24 \mathrm{~h}$ of biofilm growth, slabs were treated 8 times/day, 3 days for dentine and 4 days for enamel. The culture medium was changed twice, before the first treatment of the day and after the last treatment of the day. The $\mathrm{pH}$ of each change of medium was measured as indicator of biofilm acidogenicity.

\section{Treatments (Figure 1)}

The carbohydrate solutions were the same as those previously used..$^{10}$ The starch solution was prepared from soluble starch (S9765, 80\% amylopectin and 20\% amylose; Sigma Chemical Co., St. Louis, USA), and the sucrose solution was prepared from powdered sucrose (107651, Merck Millipore, Darmstadt, Germany). To prepare $1 \%$ starch and $1 \%$ starch $+10 \%$ sucrose, the suspensions were boiled until dissolution was complete. All solutions were autoclaved and stored at room temperature. During the experiments, the solutions were aseptically transferred to the 24-well plates to be used.

The biofilms on the enamel and dentine slabs were individually treated 8 times/day at defined times (9:00, 10:30, 12:00, 13:00, 14:30, 16:00, 17:00, and 18:30 h). Before each treatment, the slabs were removed from the UTYEB medium containing $0.1 \mathrm{mM}$ glucose and $10 \%$ saliva and transferred to a new plate containing saliva for the pretreatment. After $1 \mathrm{~min}$, they were transferred to another plate containing the specified treatments $(0.9 \% \mathrm{NaCl}$, $1 \%$ starch, $10 \%$ sucrose, or $1 \%$ starch $+10 \%$ sucrose). After $3 \mathrm{~min}$, the slabs were washed 3 times with $0.9 \% \mathrm{NaCl}$ and returned to the culture plate containing the medium described above.

\section{Biofilm collection and analysis}

After 4 and 5 days of biofilm growth for dentine and enamel, respectively, ${ }^{16,17}$ the slabs were individually washed 3 times with $0.9 \% \mathrm{NaCl}$, transferred to microcentrifuge tubes containing $1 \mathrm{~mL}$ of $0.9 \% \mathrm{NaCl}$, and sonicated for $30 \mathrm{~s}$ at $7 \mathrm{~W}$ (Branson, Sonifier 150, Danbury, USA) to detach the biofilm from the slabs. ${ }^{15}$ Slabs were separated and stored for demineralization analysis, and aliquots of the suspension were analyzed for the following dependent variables. 


\section{Biomass}

Aliquots $(150 \mu \mathrm{l})$ of the suspension were centrifuged (10 min at 5,000 $\mathrm{g}$ and $4^{\circ} \mathrm{C}$ ); the pellets were dried in a Speed-Vac concentrator (Savant Instruments Inc., Hicksville, USA) for $2 \mathrm{~h}$, and then the pellets were weighed $( \pm 0.01 \mathrm{mg})$ to obtain the biofilm dry weight, which was used as a biomass indicator.

\section{Viable microorganisms}

Aliquots $(100 \mu \mathrm{l})$ of the suspension were serially diluted in $0.09 \% \mathrm{NaCl}$ and then used to inoculate $\mathrm{BHI}$ agar (BD, Sparks, USA), in triplicate, to determine the number of viable microorganisms. ${ }^{19}$ The plates were incubated for $48 \mathrm{~h}$ at $37^{\circ} \mathrm{C}$ and $10 \% \mathrm{CO}_{2}$. Colonies of S. mutans were counted and expressed as the number of CFU/mg of biofilm dry weight.

\section{Polysaccharides}

Aliquots $(400 \mu \mathrm{l})$ of the suspension were used to extract the polysaccharides and determine the concentrations of soluble polysaccharides (SEPS), insoluble extracellular polysaccharides (IEPS), EPS, and intracellular polysaccharides (IPS) in the biofilm. ${ }^{12}$ The results were normalized by biofilm dry weight and expressed as micrograms per milligram of biomass.

\section{Enamel and dentine demineralization}

The final $\mathrm{SH}$ of each slab was measured a second time using 3 indentations $100 \mu \mathrm{m}$ apart from the initial indentations or in the center of the slabs if the initial indentations were not visible. Baseline and final values were used to obtain the percentage of surface hardness loss - \%SHL: ((baseline SH value - final SH value $) \times(100 /$ baseline $S H$ value $)$, which was used as an indicator of ename ${ }^{20}$ and dentine ${ }^{21}$ demineralization.

\section{Statistical analysis}

The assumptions of equality of variances and normal distribution of errors were checked using Shapiro-Wilk's test for all response variables tested. Variables that did not satisfy these assumptions were transformed and analyzed using an analysis of variance followed by Tukey's test. Enamel and dentine data were analyzed separately. SAS 9.0 software (SAS Institute, Cary, USA) was used to perform the analyses, with a significance level fixed at $5 \%$.

\section{Results}

Compared to the other groups, starch + sucrose showed a significantly $(p<0.0001)$ more pronounced decrease in medium $\mathrm{pH}$ at 32,56, 80, and $104 \mathrm{~h}$ of biofilm growth for enamel (Figure 2A) and at 32, 56 , and $80 \mathrm{~h}$ of biofilm growth for dentine (Figure 2B).

The biofilms treated with starch + sucrose did not differ from those treated with sucrose alone with respect to the variables biomass, viable bacteria, SEPS, and IPS for either enamel (Table 1) or dentine (Table 2). The IEPS produced by the biofilms treated with starch + sucrose were significantly higher $(\mathrm{p}<0.0001)$ than those by biofilms treated with sucrose alone for dentine (Table 2) but not for enamel (Table 1).
A

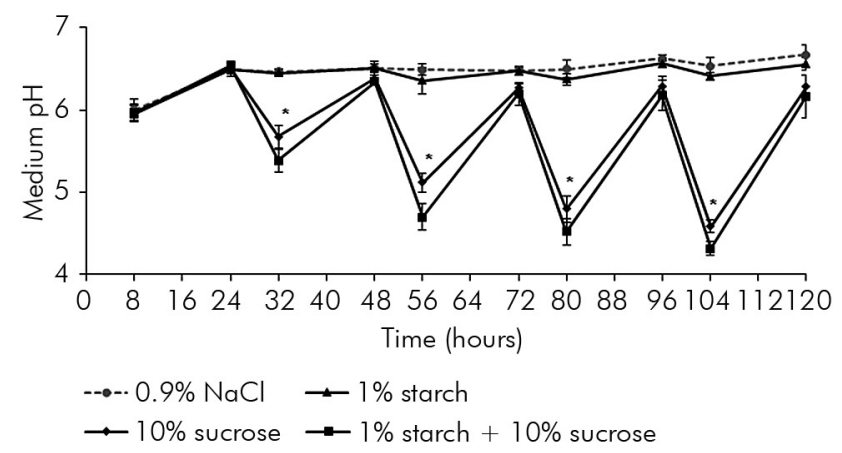

B

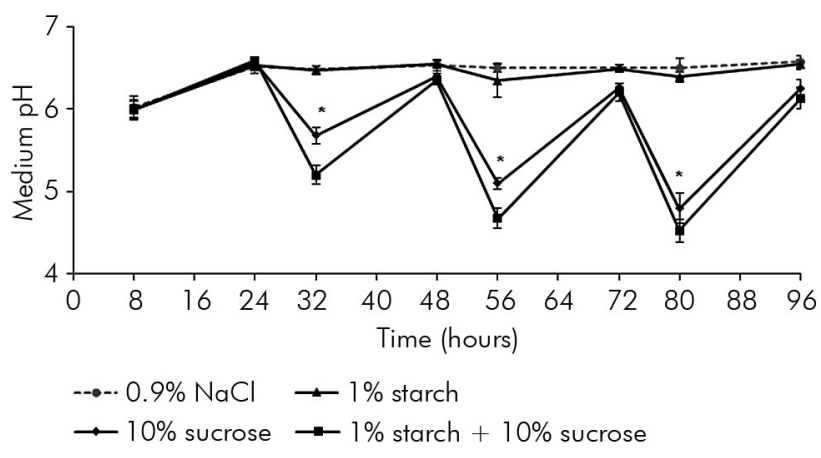

Figure 2. Acidogenicity of the biofilms (medium $\mathrm{pH}$ ) formed on enamel $(\mathrm{A})$ and dentine $(\mathrm{B})$ slabs $(\mathrm{mean} \pm \mathrm{SD}, \mathrm{n}=9)$ according to time $(\mathrm{h})$ and treatment $(0.9 \% \mathrm{NaCl}, 1 \%$ starch, $10 \%$ sucrose, $1 \%$ starch $+10 \%$ sucrose). * Significant differences between starch + sucrose and sucrose group $(p<0.0001)$. 
Table 1. Composition of biofilms formed on enamel subjected to the indicated treatments (mean $\pm S D, n=9$ )

\begin{tabular}{|c|c|c|c|c|c|}
\hline \multirow[b]{2}{*}{ Treatments } & \multicolumn{5}{|c|}{ Dependent variables } \\
\hline & Biomass $(\mathrm{mg})^{*}$ & $\begin{array}{l}\text { Viable bacteria (CFU/mg } \\
\text { dry weight) } \times 10^{9 *}\end{array}$ & $\begin{array}{l}\text { SEPS }(\mu \mathrm{g} / \mathrm{mg} \text { dry } \\
\text { weight)* }\end{array}$ & $\begin{array}{l}\text { IEPS }(\mu \mathrm{g} / \mathrm{mg} \text { dry } \\
\text { weight) }\end{array}$ & $\begin{array}{l}\text { IPS }(\mu \mathrm{g} / \mathrm{mg} \mathrm{dry} \\
\text { weight)* }\end{array}$ \\
\hline $0.9 \% \mathrm{NaCl}$ & $0.5 \pm 0.3^{a}$ & $1.9 \pm 1.6^{a}$ & $3.1 \pm 1.0^{a}$ & $2.5 \pm 1.2^{a}$ & $2.6 \pm 2.0^{a}$ \\
\hline $1 \%$ starch & $0.5 \pm 0.2^{a}$ & $1.9 \pm 0.9^{a}$ & $3.01 \pm 1.2^{a}$ & $2.7 \pm 1.1^{\mathrm{a}}$ & $4.1 \pm 2.0^{a}$ \\
\hline $10 \%$ sucrose & $1.8 \pm 0.4^{b}$ & $1.8 \pm 0.4^{a}$ & $2.21 \pm 0.9^{a}$ & $23.1 \pm 13.3^{b}$ & $2.8 \pm 1.2^{a}$ \\
\hline $1 \%$ starch $+10 \%$ sucrose & $1.7 \pm 0.5^{b}$ & $1.8 \pm 1.1^{\mathrm{a}}$ & $2.20 \pm 0.5^{a}$ & $26.7 \pm 14.7^{b}$ & $4.1 \pm 1.2^{a}$ \\
\hline
\end{tabular}

SEPS, soluble extracellular polysaccharides; IEPS, insoluble extracellular polysaccharides; IPS, intracellular polysaccharides

${ }^{*}$ For statistical analysis, biomass was transformed using the square root; Viable bacteria was transformed using $(X)^{-2}$; SEPS, IEPS, and IPS were transformed using $\log _{10}(X)$. Within columns, distinct letters indicate significant differences among the treatment groups $(p<0.0001)$.

Table 2. Composition of biofilms formed on dentine subjected to the indicated treatments (mean $\pm S D, n=9$ )

\begin{tabular}{|c|c|c|c|c|c|}
\hline \multirow[b]{2}{*}{ Treatments } & \multicolumn{5}{|c|}{ Dependent variables } \\
\hline & Biomass $(\mathrm{mg})^{*}$ & $\begin{array}{c}\text { Viable bacteria (CFU/mg } \\
\text { dry weight) } \times 10^{9 *}\end{array}$ & $\begin{array}{l}\text { SEPS }(\mu \mathrm{g} / \mathrm{mg} \text { dry } \\
\text { weight)* }\end{array}$ & $\begin{array}{l}\text { IEPS }(\mu \mathrm{g} / \mathrm{mg} d r y \\
\text { weight)* }\end{array}$ & $\begin{array}{l}\text { IPS }(\mu \mathrm{g} / \mathrm{mg} d r y \\
\text { weight) }\end{array}$ \\
\hline $0.9 \% \mathrm{NaCl}$ & $0.6 \pm 0.2^{a}$ & $1.3 \pm 0.6^{a}$ & $3.2 \pm 2.7^{a}$ & $2.7 . \pm 1.3^{a}$ & $5.2 \pm 2.1^{a * *}$ \\
\hline $1 \%$ starch & $0.6 \pm 0.2^{a}$ & $2.8 \pm 1.0^{b}$ & $3.4 \pm 1.9^{a}$ & $5.7 \pm 3.5^{a}$ & $5.9 \pm 1.5^{a}$ \\
\hline $10 \%$ sucrose & $1.3 \pm 0.4^{b}$ & $2.6 \pm 0.8^{b}$ & $3.1 \pm 1.5^{\mathrm{a}}$ & $20.3 \pm 3.8^{b}$ & $4.3 \pm 1.3^{a}$ \\
\hline $1 \%$ starch $+10 \%$ sucrose & $1.2 \pm 0.4^{b}$ & $2.0 \pm 0.3^{b}$ & $2.5 \pm 1.2^{a}$ & $28.7 \pm 7.0^{c}$ & $5.3 \pm 1.5^{a}$ \\
\hline
\end{tabular}

SEPS, soluble extracellular polysaccharides; IEPS, insoluble extracellular polysaccharides; IPS, intracellular polysaccharides

${ }^{*}$ For statistical analysis, biomass and IEPS were transformed using $\log _{10}(X)$; viable bacteria were transformed using $(X)^{-2}$; SEPS were transformed using the square root.

**One value indicated by the SAS software to be an outlier (1 1.36) was removed. Within the columns, distinct letters indicate significant differences among the treatment groups $(p<0.0001)$.

Regarding demineralization, treatment using starch + sucrose caused significantly $(\mathrm{p}<0.0001)$ greater \%SHL both for enamel and dentine (Figure 3 ) in comparison with treatment using sucrose alone.

\section{Discussion}

Starch and sucrose make up the largest proportion of dietary carbohydrates consumed worldwide. ${ }^{4}$ While some studies have reported that starch + sucrose is more cariogenic than sucrose alone, ${ }^{8,9,10}$ others have found no difference. ${ }^{11,12}$

Our results showed higher demineralization of bovine enamel and dentine when they were exposed to starch + sucrose than when they were exposed to sucrose alone (Figure 3). With respect to enamel, our results are in agreement with those found in situ for deciduous enamel.$^{10}$ However, they contrast with the in vitro results reported by Thurnheer et al., ${ }^{11}$ using a multispecies biofilm model. This disagreement could be because of the different biofilm models used and how the biofilms were exposed to carbohydrates. In our study, exposure of the biofilm to carbohydrates was intermittent (8 times/day), whereas Thurnheer et al. ${ }^{11}$ exposed the biofilm to carbohydrate using continuous

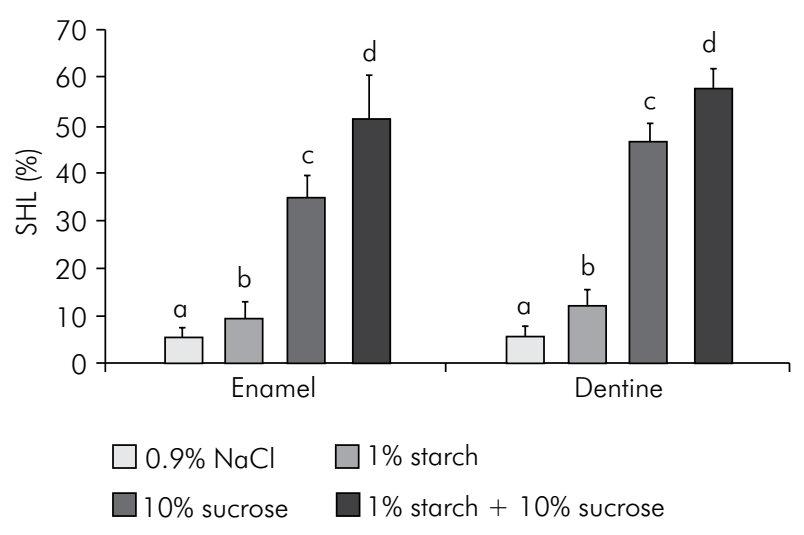

Figure 3. Percentage of surface hardness loss (\%SHL) in enamel and dentine slabs according to the treatments administered to the biofilms (mean $\pm S D, n=9$ ). Different letters indicate significant differences $(\mathrm{p}<0.0001)$ among treatments (within the dental substrates). For statistical analysis, $\%$ SHL for enamel was transformed using the square root. 
feeding in culture medium. Consequently, medium $\mathrm{pH}$ was kept at values below 5.0 for all treatments, while in our study, it was possible to show differences in acidogenicity among the treatments (Figure 2).

With regard to dentine, our study showed that starch + sucrose caused greater demineralization than sucrose alone (Figure 3). These results apparently disagree with those found in situ ${ }^{12}$ and could not be explained by differences in substrate, since both studies used bovine root dentine. Indeed, Aires et al. ${ }^{12}$ observed a trend toward a greater effect for starch + sucrose than for sucrose alone, but the difference was not statistically significant. In addition to the inherent differences in ability to control variables between in vitro and in situ studies, the volunteers were exposed to fluoride from water and dentifrice in the in situ study above, which could have masked the cariogenic potential of starch + sucrose. The present in vitro study was conducted in the absence of fluoride.

The more pronounced effect of starch + sucrose on the demineralization of both enamel and dentine may not be attributed to the fact that the final concentration of carbohydrate in the starch + sucrose mixture was $11 \%$ ( $1 \%$ starch $+10 \%$ sucrose), while it was $10 \%$ for sucrose alone. Indeed, we conducted a complementary study comparing the effect of $1 \%$ starch $+9 \%$ sucrose versus $1 \%$ starch $+10 \%$ sucrose, and the difference in enamel demineralization between them was not statistically significant (data not shown).

Therefore, the effect of starch + sucrose on the demineralization of enamel and dentine may be considered synergistic and not simply the sum of the effect of fermentation of $1 \%$ starch and $10 \%$ sucrose. The data show that starch caused an enamel SHL that was 4.1\% higher than that of the control, while for sucrose, this figure was $29.5 \%$ (Figure 3 ). If the demineralization were the sum of these effects, a $33.6 \%$ greater demineralization would have been expected for the starch + sucrose group, compared with the control. However, the effect of the combination was $45.9 \%$ higher, increasing the cariogenic effect of sucrose by 1.4-fold. For dentine, the sum of the effect of carbohydrates alone was $47.5 \%$, while the effect of the combination was $52.1 \%$. The increased effect is supported by the statistical analysis, which showed that the effect of starch + sucrose was greater than the effect of the carbohydrates separately (Figure 3).
This enhanced effect of starch + sucrose on enamel and dentine demineralization was confirmed by the acidogenicity data (Figure 2A and B). When biofilms were exposed to starch + sucrose, the concentration of $\mathrm{H}^{+}$in the medium at $32,56,80$, and $104 \mathrm{~h}$ of biofilm growth was higher than that found when the biofilms were exposed to the carbohydrates separately. For example, at $56 \mathrm{~h}$, the $\mathrm{H}^{+}$concentrations for the groups treated with starch and sucrose separately were $1.2 \times 10^{-7}$ and $74.2 \times 10^{-7} \mathrm{M}$, respectively. However, the value found for the group treated with starch + sucrose was $200.9 \times 10^{-7} \mathrm{M}$, which is 2.7 -fold higher than the sum of the effects of starch and sucrose separately.

While explaining this increased effect was not an aim of the present study, it could be the result of increased starch degradation to fermentable products by amylase present in S. mutans. It is known that the action of this enzyme is essential for starch to be metabolized by bacteria present in biofilms, ${ }^{13}$ mainly S. mutans, which do not have amylolytic activity. ${ }^{22}$ This enzyme is found in acquired pellicle ${ }^{23}$ and in biofilm matrix. ${ }^{14}$ In our biofilm model, the action of this enzyme in both sites was provided by pretreatment of the dental substrates with saliva and by the presence of saliva in the culture medium in which the biofilms were grown (see Methodology). It is well known that for any carbohydrate to be fermented by a bacterial biofilm, the carbohydrate must first diffuse into the biofilm matrix and be transferred to the bacterial cytoplasm. However, this process is hampered when starch is used as the carbohydrate source for the biofilm bacteria, since its diffusion into the biofilm is limited because of its high molecular weight. ${ }^{24}$ In addition, it must first be degraded in the biofilm matrix to form products than can be transported into the bacteria. ${ }^{25}$ However, this diffusion can be facilitated by the effect of sucrose and starch on the matrix of the biofilm formed. ${ }^{5}$

Thus, the concentration of IEPS in the biofilm exposed to starch + sucrose was greater than that found in the biofilm exposed to starch alone (Tables 1 and 2). Compared with the group treated only with sucrose, starch + sucrose showed a significantly higher concentration of IEPS in the biofilms grown on dentine (Table 2). It is well known that sucrose changes the biofilm matrix composition, ${ }^{2}$ making the biofilm more porous. ${ }^{26}$ It has also been suggested 
that EPS produced by sucrose in the presence of starch hydrolysates have a differentiated structure, which could explain the higher cariogenicity of starch + sucrose compared with sucrose alone. ${ }^{27,28}$ These results could be explained by increased starch diffusion into the biofilm exposed to starch + sucrose. Once inside the biofilm, the starch might be hydrolyzed by amylase to products that can be fermented by S. mutans.

The findings showing that starch + sucrose is more cariogenic than sucrose alone are supported by prospective cohort studies suggesting that the consumption of processed or cooked starches with sucrose was associated with a greater caries incidence in children and adolescents. ${ }^{29,30}$

However, in the present study, the increased cariogenicity found for starch + sucrose, compared with sucrose alone, could be because of some uncontrolled factor. The lower $\mathrm{pH}$ observed in the medium where biofilms treated with starch + sucrose were maintained, compared with the medium where biofilms were treated with sucrose alone (Figure $1 \mathrm{~A}$ and $\mathrm{B}$ ), could be because of a contamination of the medium by sugars, even after washing 3 times with $0.9 \% \mathrm{NaCl}$, mainly considering the high viscosity of starch. We checked this possibility and found it to be irrelevant because the residual concentration of sugar found in the medium was very low $(0.03 \%)$. Indeed, the $\mathrm{pH}$ of the medium after the $8^{\text {th }}$ exposure to the treatments, when the biofilms were immediately transferred to fresh medium and maintained overnight in fresh medium (times 24, 48, 72, and $96 \mathrm{~h}$ ), did not show a difference between the sucrose and starch + sucrose groups.

\section{References}

1. Fejerskov O. Changing paradigms in concepts on dental caries: consequences for oral health care. Caries Res. 2004;38(3):182-91. doi:10.1159/000077753

2. Paes Leme AF, Koo H, Bellato CM, Bedi G, Cury JA. The role of sucrose in cariogenic dental biofilm formation--new insight. J Dent Res. 2006;85(10):878-87. doi:10.1177/154405910608501002

3. Sheiham A. Dietary effects on dental diseases. Public Health Nutr. 2001;4(2B):569-91. doi:10.1079/PHN2001142

4. Lingström P, van Houte J, Kashket S. Food starches and dental caries. Crit Rev Oral Biol Med. 2000;11(3):366-80. doi:10.1177/10454411000110030601
Another limitation of the present study was the use of a biofilm model based on S. mutans, a bacterium that is unable to metabolize starch. ${ }^{22}$ Therefore, we improved our model by adding human saliva and allowing the starch to be degraded by salivary amylase. The starch was degraded by salivary amylase to form products fermentable by S. mutans, which was confirmed by the acidogenicity of the culture medium (Figure 2A and B) and by the demineralization of enamel and dentine among the groups treated with starch (Figure 3). Therefore, further studies are needed to investigate the cariogenicity of starchy products, particularly combinations of starch and sucrose, using more a specific biofilm model. This model should include S. mutans, the most cariogenic bacterium, and other bacteria that are able to metabolize starch and to adsorb salivary amylase. Studies in this direction were already conducted with 3-species biofilm composed by A. naeslundii, S. gordonii, and S. mutans. ${ }^{31}$

\section{Conclusion}

In summary, we showed that starch increases the cariogenic potential of sucrose in a S. mutans biofilm model.

\section{Acknowledgments}

The authors thank Dr. Wander José da Silva for assistance in statistical analysis. This study was supported by Conselho Nacional de Desenvolvimento Científico e Tecnológico - CNPq (no. 475178/2011-4 and no. 305310/2011-9) and Fundação de Desenvolvimento da Unicamp - FUNCAMP (Conv. 65/91 and 4252).

5. Bowen WH, Koo H. Biology of Streptococcus mutans-derived glucosyltransferases: role in extracellular matrix formation of cariogenic biofilms. Caries Res. 2011;45(1):69-86. doi:10.1159/000324598

6. Duarte S, Klein MI, Aires CP, Cury JA, Bowen WH, Koo H. Influences of starch and sucrose on Streptococcus mutans biofilms. Oral Microbiol Immunol. 2008;23(3):206-12. doi:10.1111/j.1399-302X.2007.00412.x

7. Klein MI, Duarte S, Xiao J, Mitra S, Foster TH, Koo H. Structural and molecular basis of the role of starch and sucrose in Streptococcus mutans biofilm development. Appl Environ Microbiol. 2009;75(3):837-41. doi:10.1128/AEM.01299-08 
8. Firestone AR, Schmid R, Mühlemann HR. Cariogenic effects of cooked wheat starch alone or with sucrose and frequency-controlled feedings in rats. Arch Oral Biol. 1982;27(9):759-63. doi:10.1016/0003-9969(82)90026-7

9. Mundorff-Shrestha SA, Featherstone JD, Eisenberg AD, Cowles E, Curzon ME, Espeland MA, et al. Cariogenic potential of foods. II. Relationship of food composition, plaque microbial counts, and salivary parameters to caries in the rat model. Caries Res. 1994;28(2):106-15. doi:10.1159/000261630

10. Ribeiro CCC, Tabchoury CPM, Del Bel Cury AA, Tenuta LMA, Rosalen PL, Cury JA. Effect of starch on the cariogenic potential of sucrose. Br J Nutr. 2005;94(1):44-50. doi:10.1079/BJN20051452

11. Thurnheer T, Giertsen E, Gmür R, Guggenheim B. Cariogenicity of soluble starch in oral in vitro biofilm and experimental rat caries studies: a comparison. J Appl Microbiol. 2008;105(3):829-36. doi:10.1111/j.1365-2672.2008.03810.x

12. Aires CP, Del Bel Cury AA, Tenuta LMA, Klein MI, Koo H, Duarte S, et al. Effect of starch and sucrose on dental biofilm formation and on root dentine demineralization. Caries Res. 2008;42(5):380-6. doi:10.1159/000154783

13. Scannapieco FA, Torres G, Levine MJ. Salivary alpha-amylase: role in dental plaque and caries formation. Crit Rev Oral Biol Med. 1993 Jan;4(3-4):301-7. doi:10.1177/10454411930040030701

14. Fiehn NE, Moe D. Alpha-amylase activity in supragingival dental plaque in humans. Scand J Dent Res. 1983 Oct;91(5):365-70. doi:10.1111/j.1600-0722.1983.tb00831.x

15. Ccahuana-Vásquez RA, Cury JA. S. mutans biofilm model to evaluate antimicrobial substances and enamel demineralization. Braz Oral Res. 2010;24(2):135-41. doi:10.1590/S1806-83242010000200002

16. Muñoz-Sandoval C, Muñoz-Cifuentes MJ, Giacaman RA, Ccahuana-Vasquez RA, Cury JA. Effect of bovine milk on Streptococcus mutans biofilm cariogenic properties and enamel and dentin demineralization. Pediatr Dent. 2012 Nov-Dec;34(7):E197-201.

17. Giacaman RA, Muñoz MJ, Ccahuana-Vasquez RA, Muñoz-Sandoval C, Cury JA. Effect of fluoridated milk on enamel and root dentin demineralization evaluated by a biofilm caries model. Caries Res. 2012;46(5):460-6. doi:10.1159/000339428

18. Koo H, Vacca-Smith AM, Bowen WH, Rosalen PL, Cury JA, Park YK. Effects of Apis mellifera propolis on the activities of streptococcal glucosyltransferases in solution and adsorbed onto saliva-coated hydroxyapatite. Caries Res. 2000;34(5):418-26. doi:10.1159/000016617

19. Herigstad B, Hamilton M, Heersink J. How to optimize the drop plate method for enumerating bacteria. J Microbiol Methods. 2001;44(2):121-9. doi:10.1016/S0167-7012(00)00241-4
20. Cury JA, Rebelo MA, Del Bel Cury AA, Derbyshire MT, Tabchoury CPM. Biochemical composition and cariogenicity of dental plaque formed in the presence of sucrose or glucose and fructose. Caries Res. 2000;34(6):491-7. doi:10.1159/000016629

21. Vale GC, Tabchoury CPM, Del Bel Cury AA, Tenuta LMA, ten Cate JM, Cury JA. APF and dentifrice effect on root dentin demineralization and biofilm. J Dent Res. 2011;90(1):77-81. doi:10.1177/0022034510383428

22. Edwardsson S. Characteristics of caries-inducing human streptococci resembling Streptococcus mutans. Arch Oral Biol. 1968;13(6):637-46. doi:10.1016/0003-9969(68)90142-8

23. Hannig C, Attin T, Hannig M, Henze E, Brinkmann K, Zech R. Immobilisation and activity of human alpha-amylase in the acquired enamel pellicle. Arch Oral Biol. 2004;49(6):469-75. doi:10.1016/j.archoralbio.2004.01.005

24. Thurnheer T, Gmür R, Shapiro S, Guggenheim B. Mass transport of macromolecules within an in vitro model of supragingival plaque. Appl Environ Microbiol. 2003;69(3):1702-9. doi:10.1128/AEM.69.3.1702-1709.2003

25. Webb AJ, Homer KA, Hosie AHF. Two closely related $\mathrm{ABC}$ transporters in Streptococcus mutans are involved in disaccharide and/or oligosaccharide uptake. J Bacteriol. 2008;190(1):168-78. doi:10.1128/JB.01509-07

26. Dibdin GH, Shellis RP. Physical and biochemical studies of Streptococcus mutans sediments suggest new factors linking the cariogenicity of plaque with its extracellular polysaccharide content. J Dent Res. 1988;67(6):890-5. doi:10.1177/00220345880670060101

27. Vacca-Smith AM, Venkitaraman AR, Quivey RG Jr, Bowen WH. Interactions of streptococcal glucosyltransferases with alpha-amylase and starch on the surface of saliva-coated hydroxyapatite. Arch Oral Biol. 1996;41(3):291-8. doi:10.1016/0003-9969(95)00129-8

28. Xiao J, Koo H. Structural organization and dynamics of exopolysaccharide matrix and microcolonies formation by Streptococcus mutans in biofilms. J Appl Microbiol. 2010;108(6):2103-13. doi:10.1111/j.1365-2672.2009.04616.x

29. Chankanka O, Marshall TA, Levy SM, Cavanaugh JE, Warren JJ, Broffitt B, et al. Mixed dentition cavitated caries incidence and dietary intake frequencies. Pediatr Dent. 2011;33(3):233-40.

30. Campain AC, Morgan MV, Evans RW, Ugoni A, Adams GG, Conn JA, et al. Sugar-starch combinations in food and the relationship to dental caries in low-risk adolescents. Eur J Oral Sci. 2003;111(4):316-25. doi:10.1034/j.1600-0722.2003.00056.x

31. Cavalcanti YW, Bertolini MM, Silva WJ, Del Bel Cury AA, Tenuta LMA, Cury JA. A three-species biofilm model for the evaluation of enamel and dentin demineralization. Biofouling. 2014;30(5):579-88. doi:10.1080/08927014.2014.905547 